\title{
The role of behavioural science in changing deprescribing practice
}

Over $50 \%$ of older people are prescribed a medicine with more harm than benefit leading to avoidable morbidity, hospitalisation and mortality(1). The World Health Organisation has recognised this problem in its recent Global Patient Safety Challenge: Medication Without Harm. Deprescribing is the process of identifying and discontinuing medicines that are no longer appropriate. The term deprescribing is a relative newcomer to the research and clinical vocabulary. Medicines may be deprescribed for several reasons including when they are no longer indicated, when the harms outweigh the benefits or when treatment may not align with the patient's healthcare goals and treatment preferences. Whilst the principle of deprescribing has always been an expectation of good prescribing practice, there are numerous barriers to it becoming routine that are discussed in section $3(2,3)$.

Deprescribing involves establishing an accurate account of the patient's prescribed medicines, identifying medicines that are appropriate for deprescribing, achieving agreement with the patient to attempt deprescribing and ensuring monitoring and appropriate safety netting are in place. It therefore requires practitioner and patient behaviours to align as they collaboratively navigate the process. Despite global traction towards developing strategies to support practitioners and patients to deprescribe, there have been only marginal successes in effecting change in deprescribing behaviour(4).

The three key unknowns to designing interventions to change behaviour are: 1 . defining the target behaviour; 2 . specifying whose behaviour needs to change and 3. identifying how to achieve the desired change(3). The field of behavioural science applies theory and empirical evidence to address these three unknowns.

\section{Defining the target behaviour}

We have often fallen at the first hurdle when defining the target behaviour in deprescribing; some define it as stopping medicines to prevent harm, others consider it to be stopping medicines in response to harm and some fail to make any distinction. The terms 'reactive deprescribing' and 'proactive deprescribing' emerged in 2018 to define these two distinct behaviours(5). Reactive deprescribing is "discontinuing a medicine in response to an adverse clinical trigger". Proactive deprescribing is "discontinuing a medicine if future gains are unlikely to outweigh future harms". The barriers and enablers to each behaviour differ substantially. Practitioner motivation to reactively deprescribe is likely to be very high given that failure to undertake the behaviour leads to certain patient harm(2). For example, reactively deprescribing anticoagulant for a patient presenting with a gastric bleed. Motivation to proactively deprescribe, however, may be hampered by the uncertainties regarding the likely benefits and potential harms(3). For example, a practitioner may be reticent to proactively deprescribe an antihypertensive for a patient even if the risk of falling and resulting fracture is greater than the risk of a cardiovascular event. This difference is reflected in practice with $80 \%$ of all medicines deprescribed in hospital being reactive. The $20 \%$ of proactive deprescribing cases all had documented evidence of no clinical benefit thus only potential for harm, rather than a complex evaluation of risks and benefits. Proactive deprescribing is therefore the behaviour that requires targeting to reduce avoidable medication-related harm. 


\section{Specifying whose behaviour needs to change}

Both practitioner and patient behaviour are key to successful proactive deprescribing and interventions targeting both have been developed. However, patient behaviour change interventions have only been reported for medicines where guidelines specify that continued prescribing is inappropriate such as the EMPOWER brochure. This provides patients with information regarding the risks of benzodiazepines which guidelines state should not be continued long-term for the management of insomnia. The EMPOWER brochure encourages patients to initiate deprescribing conversations with healthcare practitioners. It was tested in a randomised controlled trial with community-dwelling individuals and demonstrated that $27 \%$ of patients had a benzodiazepine deprescribed at six months, which was $22 \%$ greater than the control(7). For medicines with less clarity regarding when to deprescribe, patients and carers want these deprescribing discussions to be initiated by the prescriber(6). Over $80 \%$ of patients and carers indicate they would be willing to have a medicine deprescribing if this was recommended by their prescriber(6).

There is a growing body of literature to support practitioners in screening medicines for potential deprescribing such as Beers and STOPP, and guidelines directing how to safely discontinue medicines have also been developed(8). However, a systematic review of randomised controlled trials of practitioner focussed deprescribing interventions comprising primarily screening tools alone, resulted in a mean effect size of less than one medicine deprescribed(4) indicating that addressing knowledge and skills alone is insufficient. Moving forward, the focus should therefore be on developing practitioner behaviour change interventions that incorporate the other components to address the remaining barriers and enablers. These interventions should support practitioners to work with patients to facilitate shared deprescribing decision-making.

\section{Identifying how to achieve the desired change}

Behaviour change interventions should address the barriers and enablers (determinants) from the perspective of the person whose behaviour requires changing(9). Behaviour change theory provides the lens through which these determinants can be conceptualised and appropriate behaviour change techniques (BCTs) selected. BCTs are the 'active ingredients' of interventions that address the determinants to bring about the required change in behaviour(9).

The determinants of deprescribing from the primary care practitioner perspective were reported in a 2014 systematic review(2). Limited skills, knowledge and perceived patient resistance to deprescribing are key barriers, whereas access to specialists in deprescribing such as geriatricians and pharmacists for advice is an enabler. Clinical guidelines are also heavy influencers of prescribing practice, and the current overrepresentation of single-disease guidelines that focus solely on prescribing rather than deprescribing are a barrier $(2,3)$.

Application of behaviour change theory to develop proactive deprescribing interventions to address the determinants in primary care is yet to be reported(2).The hospital deprescribing implementation framework (hDIF) provides the five key determinants to proactive deprescribing in hospital and 44 BCTs for addressing these determinants(3). The hDIF affords the flexibility of each of the five determinants being linked to between five and 17 BCTs. This enables organisations to select from these BCTs according to individual context such as organisational resources. This flexibility offered by the hDIF aligns the new way of working with the target audience, gains trust and promotes ownership(3). Selection of BCTs from the hDIF in partnership with the target audience of geriatricians and pharmacists was recently reported for the UK hospital context. Selected BCTs 
include organisational action planning to raise the priority of deprescribing in hospitals, restructuring pharmacists' working patterns to enable them to attend multidisciplinary team meetings and measuring, reporting and sharing of deprescribing activity between teams to incentivise the activity(10).A programme of work to test the intervention is now underway to determine effectiveness and cost-effectiveness in changing practitioner proactive deprescribing behaviour.

As the body of trial evidence testing practitioner deprescribing behaviour change interventions builds, there comes a need to develop and validate measures of success. The extent to which the intervention leads to the desired behaviour change as measured by the number of medicines deprescribed determines efficacy. Whilst this is clearly an important process measure, patient and health system orientated measures are necessary for informing policy and commissioning decisions.

Core outcome sets standardise the outcomes that should be measured and reported for the specific area of health under investigation. There is currently no core outcome set for trials of practitioner deprescribing interventions. Cost and quality of life are central to most medication related core outcome sets and are likely to be incorporated into a deprescribing core outcome set. Additionally, the patient experience of the care delivered by the practitioner is also an important component of most core outcome sets. Whilst the practitioner is the recipient of the behaviour change intervention, the patient is the recipient of the resulting practitioner behaviour. A key measure of success is therefore a positive patient experience. For example, this may culminate in patients feeling that medicines have been comprehensively reviewed by the prescribed, that they have been adequately supported to make shared decisions and that they have confidence in the ongoing monitoring strategy. Developing and validating a tool to capture the patient experience will therefore serve to both evaluate and inform necessary refinements of the intervention.

\section{Conclusion}

Proactive deprescribing is an essential weapon in our armoury against inappropriate medication use. However, it is a well-recognised deficit in clinical practice. Patients want practitioners to initiate the process, yet over a decade of research endeavour has been divided between practitioner and patient behaviour change. This may explain the marginal progress in achieving the required practitioner behaviour change. The boom in deprescribing research is therefore here to stay with an additional focus on practitioner behaviour change to complement ongoing patient behaviour change research. Capturing the patient experience should also become a central component in the evaluation of these deprescribing behaviour change interventions.

\section{References}

1. Gallagher P, Lang PO, Cherubini A, Topinková E, Cruz-Jentoft A, Montero Errasquín B, et al. Prevalence of potentially inappropriate prescribing in an acutely ill population of older patients admitted to six European hospitals. Eur J Clin Pharmacol. 2011;67(11):1175-88.

2. Anderson K, Stowasser D, Freeman C, Scott I. Prescriber barriers and enablers to minimising potentially inappropriate medications in adults: a systematic review and thematic synthesis. BMJ Open. 2014;4(12):e006544.

3. Scott S, Twigg MJ, Clark A, Farrow C, May H, Patel M, et al. Development of a hospital deprescribing implementation framework: $A$ focus group study with geriatricians and pharmacists. Age Ageing. 2020;49(1):102-10. 
4. Page AT, Clifford RM, Potter K, Schwartz D, Etherton-Beer CD. The feasibility and effect of deprescribing in older adults on mortality and health: a systematic review and meta-analysis. Br J Clin Pharmacol. 2016;82(3):583-623.

5. Scott S, Clark A, Farrow C, May H, Patel M, Twigg MJ, et al. Deprescribing admission medication at a UK teaching hospital; a report on quantity and nature of activity. Int J Clin Pharm. 2018;

6. Scott S, Clark A, Farrow C, May H, Patel M, Twigg MJ, et al. Attitudinal predictors of older peoples' and caregivers' desire to deprescribe in hospital. BMC Geriatr. 2019;19(1):108.

7. Tannenbaum C, Martin P, Tamblyn R, Benedetti A, Ahmed S. Reduction of inappropriate benzodiazepine prescriptions among older adults through direct patient education: The EMPOWER cluster randomized trial. JAMA Intern Med. 2014;174(6):890-8.

8. Reeve E. Deprescribing tools: a review of the types of tools available to aid deprescribing in clinical practice. J Pharm Pract Res. 2020;50(1):98-107.

9. Michie S, van Stralen MM, West R, Grimshaw J, Shirran L, Thomas R, et al. The behaviour change wheel: A new method for characterising and designing behaviour change interventions. Implement Sci [Internet]. 2011;6(1):42. Available from: http://implementationscience.biomedcentral.com/articles/10.1186/1748-5908-6-42

10. Scott S, May H, Patel M, Wright DJ, Bhattacharya D. A practitioner behaviour change intervention for deprescribing in the hospital setting. Age Ageing [Internet]. 2020 Sep 2; Available from: https://doi.org/10.1093/ageing/afaa169 


\section{Conflict of interest}

The authors have no conflicts of interest to declare. 